\title{
Critical phenomena in active matter
}

\author{
M. Paoluzzi, ${ }^{1, *}$ C. Maggi, ${ }^{2}$ U. Marini Bettolo Marconi, ${ }^{3}$ and N. Gnan ${ }^{4}$ \\ ${ }^{1}$ Department of Physics, Syracuse University, Syracuse, New York 13244, USA \\ ${ }^{2}$ NANOTEC-CNR, Institute of Nanotechnology, Soft and Living Matter Laboratory, Piazzale A. Moro 2, I-00185, Roma, Italy \\ ${ }^{3}$ Scuola di Scienze e Tecnologie, Università di Camerino, Via Madonna delle Carceri, 62032 Camerino, Italy \\ and INFN, Sezione di Perugia, Via A. Pascoli, 06123 Perugia, Italy \\ ${ }^{4}$ CNR, ISC, UOS Sapienza, Piazzale Aldo Moro 2, 00185 Roma, Italy
}

(Received 10 June 2016; published 3 November 2016)

\begin{abstract}
We investigate the effect of self-propulsion on a mean-field order-disorder transition. Starting from a $\varphi^{4} \operatorname{scalar}$ field theory subject to an exponentially correlated noise, we exploit the unified colored-noise approximation to map the nonequilibrium active dynamics onto an effective equilibrium one. This allows us to follow the evolution of the second-order critical point as a function of the noise parameters: the correlation time $\tau$ and the noise strength $D$. Our results suggest that the universality class of the model remains unchanged. We also estimate the effect of Gaussian fluctuations on the mean-field approximation finding an Ornstein-Zernike-like expression for the static structure factor at long wavelengths. Finally, to assess the validity of our predictions, we compare the mean-field theoretical results with numerical simulations of active Lennard-Jones particles in two and three dimensions, finding good qualitative agreement at small $\tau$ values.
\end{abstract}

DOI: 10.1103/PhysRevE.94.052602

\section{INTRODUCTION}

Motile cells, living bacteria, synthetic swimmers, a flock of birds, and a school of fish are only a few examples of active systems able to give rise to a plethora of fascinating phenomena that spontaneously arise from their collective behavior [1-3]. In order to reproduce and understand the emergence of cooperative dynamics in active systems, several-minimal models have been put forward, most based on self-propelled agents and hydrodynamics theories but also on rule-base models with alignment interactions [4-7]. In spite of their minimal ingredients, these model systems display a highly collective behavior that results in large-scale pattern formation [8], aggregation [9], swarming [5], off-equilibrium order-disorder transitions [7], peculiar rheological properties, and disordered arrested states [10-13]. Such a rich phenomenology shares many similarities with the collective behavior in condensed matter physics where the emergence of a cooperative dynamics is intimately related to the concept of phase transitions [14]. The analogy between the collective behavior in condensed matter and spontaneous aggregation in biological or synthetic systems suggests that a coarse-grained procedure that neglects the complexity of active agents could reproduce, at least qualitatively, the observed phenomenology [2,7].

Notable attempts in this direction have focused on specific models of isotropic self-propelled particles without aligning interactions $[9,15-18]$. The fundamental ingredient that defines these nonequilibrium models is that the random force acting on each particle is not of thermal origin, i.e., is not a Brownian noise, but is a self-propulsion force that decorrelates on a time scale $\tau$. Early theoretical approaches were based on the idea of recasting the nonequilibrium dynamics in an effective equilibrium dynamics with a density-dependent diffusion coefficient [4,9], suggesting a phase transition known as motility-induced phase separation. Following the same

\footnotetext{
*mpaoluzz@syr.edu
}

idea, some of us have recently shown that the steady-state distribution of many active particles driven by Gaussian colored noise can be mapped onto an equilibrium problem where the noise amplitude and its correlation time play the role of control thermodynamic variables [15,19-21]. In that study, the mapping to an effective equilibrium dynamics was obtained due to the unified colored-noise approximation (UCNA) $[22,23]$. In addition, the random driving forces were modeled by an Ornstein-Uhlenbeck process (OUP), which gives rise to a self-propulsion that is Gaussian distributed and exponentially correlated in time. An exponentially correlated propulsion force characterizes also active Brownian [16,24] and run and tumble dynamics [25]. The OUP has been shown to model quite well the behavior of passive tracers in active suspensions [26,27]. Recently, several research groups have devoted much attention to modeling active particle systems by means of the OUP $[17,18,28]$.

Although the Gaussian colored-noise model has been analyzed at the level of few particles [15], in the case of a many-particle system it presents the same insurmountable difficulties as the equilibrium many-body problem. From this perspective, it would be desirable to develop a coarse-grained version of the model for studying phase transitions, especially to understand the effect of the memory of the noise on phase behavior. To this aim, in this article we propose and investigate a Gaussian colored-noise-driven field theory based on the UCNA. In particular, we focus on the effect of colored noise on a second-order phase transition. In this framework, we can compute the shift in the critical temperature due to the finite correlation time of the driving force. The external parameter $\tau$ changes the location of the critical point but not the universality class of the model. We find a reentrant behavior of the critical curve in the activity-noise phase diagram showing that, while for a small value of $\tau$ the phase transition is enhanced by the correlation time of the noise, at larger $\tau$ this tendency is inverted. Moreover, we compute the Gaussian fluctuations around the mean field, obtaining an Ornstein-Zernike (OZ) -like expression for the static structure 
factor at short wavelengths. The $\mathrm{OZ}$ expression predicts a power-law divergence of the correlation length at the critical point. The analytical mean-field predictions are compared with numerical simulations of a monodisperse active Lennard-Jones fluid in two and three dimensions, finding good agreement at small $\tau$ values.

\section{MODEL}

Critical phenomena are a special example of phase transitions and play a pivotal role in statistical mechanics [14,29,30]. The Landau model is the common starting point to address a phase transition. In order to extend the Landau theory to active systems, as the first step we have to fix the universality class of the problem. Without loss of generality, for the aim of this paper, we will look at scalar field theory. The scalar theory can be generalized to another universality class, i.e., we can include vectorial or tensorial fields with alignment interactions to study the emergence of nematic order [31-36].

We are interested in the case of a system close to the critical point and described by a scalar order parameter $\varphi(x)$, e.g., the magnetization in the Ising ferromagnet, or the density difference $\rho_{L}-\rho_{G}$ in the gas-liquid phase transition. The thermodynamics can be obtained by considering the equilibrium solutions of the corresponding relaxation dynamics [37]. In the case of gas-liquid transition, one should consider the model B dynamics. However, models A and B share the same static properties that are related to the Hamiltonian $H[\varphi(x)]$ as follows:

$$
\mathcal{F}(\beta)=-\frac{1}{\beta} \ln Z, \quad Z=\int \mathcal{D} \varphi(x) e^{-\beta H[\varphi(x)]},
$$

where $\beta=T^{-1}$ and $T$ is the temperature. ${ }^{1}$ To obtain the Landau-Ginzburg (LG) theory we perform the saddle-point approximation in Eqs. (1). The value $\varphi=\varphi_{S P}$ is given by the self-consistency equations

$$
\left.\frac{\delta H}{\delta \varphi(x)}\right|_{S P}=0,\left.\quad \frac{\delta^{2} H}{\delta \varphi(x)^{2}}\right|_{S P}>0
$$

and the LG free energy is $H\left[\varphi_{S P}\right]$.

\section{A. Model A with exponentially correlated noise}

In order to extend such a mean-field picture to the active counterpart we start by considering the purely dissipative dynamics of a zero-dimensional $\varphi^{4}$ scalar field theory subjected to an exponentially correlated noise. The equation of motion for the field $\varphi$ can be written in terms of an auxiliary variable $\theta$ that undergoes an OUP

$$
\begin{aligned}
& \partial_{t} \varphi(t)=-\frac{\partial H}{\partial \varphi}+\theta(t), \\
& \partial_{t} \theta(t)=-\frac{\theta(t)}{\tau}+\frac{D^{1 / 2}}{\tau} \eta(t),
\end{aligned}
$$

where the zero-mean noise $\eta$ is $\delta$ correlated, $\langle\eta(t) \eta(s)\rangle=$ $2 \delta(t-s)$, and $D$ plays the role of the (effective) temperature

\footnotetext{
${ }^{1}$ We use units such that the Boltzmann constant $k_{B}=1$.
}

of the model. The Hamiltonian $H$ is the standard $\varphi^{4}$,

$$
H[\varphi]=\frac{a}{2} \varphi^{2}+\frac{b}{4} \varphi^{4},
$$

where $a$ depends linearly on $D$ and changes sign at $D_{0}$. The coefficient $b$ is a positive constant. In the whitenoise limit, which is recovered for $\tau \rightarrow 0$, the steadystate solution of the Smoluchowski equation associated with Eq. (3) is the equilibrium distribution function $P_{e q}[\varphi] \propto \exp (-H[\varphi] / D)$.

The stochastic differential equations (3) can be rewritten as

$$
\begin{aligned}
\partial_{t}^{2} \varphi+\frac{1}{\tau} \Gamma[\varphi] \partial_{t} \varphi & =-\frac{1}{\tau} \frac{\partial H}{\partial \varphi}+\frac{D^{1 / 2}}{\tau} \eta(t), \\
\Gamma[\varphi] & \equiv 1+\tau \frac{\partial^{2} H}{\partial \varphi^{2}} .
\end{aligned}
$$

The unified colored-noise approximation is obtained by neglecting $\partial_{t}^{2} \varphi$ in Eq. (5) $[22,23]$. The corresponding Smoluchowski equation for $P[\varphi, t]$ reads [30]

$\partial_{t} P[\varphi, t]=\partial_{\varphi}\left\{\Gamma^{-1}[\varphi]\left(D \partial_{\varphi} \Gamma^{-1}[\varphi] P[\varphi, t]-\frac{\partial H}{\partial \varphi} P[\varphi, t]\right)\right\}$

and the steady-state distribution is

$$
\begin{aligned}
P_{s t}[\varphi] & =\mathcal{N} e^{-H_{\text {eff }}[\varphi] / D}, \\
H_{\text {eff }}[\varphi] & \equiv H+\frac{\tau}{2}\left(\frac{\partial H}{\partial \varphi}\right)^{2}-D \ln |\Gamma[\phi]| .
\end{aligned}
$$

From Eq. (7) it follows that $P_{s t}$ has the structure of an equilibrium distribution in terms of the effective Hamiltonian $H_{\text {eff }}$.

\section{B. Critical line}

The critical line $D_{c}(\tau)$ is the curve along which the system undergoes a second-order phase transition. In a standard $\varphi^{4}$ theory, the location of the critical point is determined by the coefficient of $\varphi^{2}$, i.e., the symmetry is spontaneously broken where $a$ changes sign. In a mean-field model described by a $L G$ free energy $\mathcal{F}_{L G}(\varphi)=a \varphi^{2} / 2+B(\varphi)$, the location of the critical point can be computed by considering the equation [30]

$$
\left.\frac{\partial^{2}}{\partial \varphi^{2}} \mathcal{F}_{L G}\right|_{\varphi=0}=a+\left.\frac{\partial^{2}}{\partial \varphi^{2}} B\right|_{\varphi=0}=0 .
$$

In our case, the LG free energy is $H_{\text {eff }}$ and the expression for $D_{c}(\tau)$ is given by

$$
\left.\frac{\partial^{2} H_{\mathrm{eff}}}{\partial \varphi^{2}}\right|_{\varphi=0}=0
$$

Now we write $a=a_{0}\left(D-D_{0}\right)$, with $a_{0}$ a positive constant. The critical curve satisfies the equation

$$
a(1+a \tau)-\frac{6 \tau b D}{1+\tau a}=0
$$



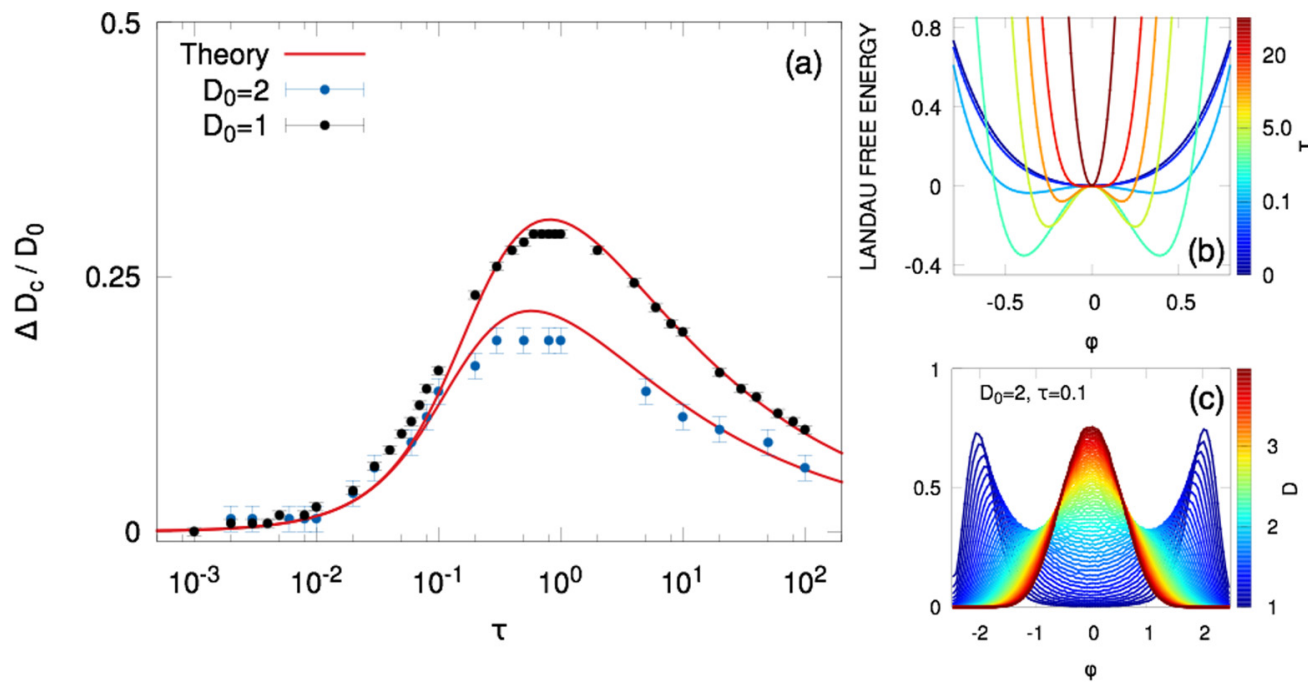

FIG. 1. Phase diagram in the activity-noise plane. (a) The red curve is obtained through Eq. (11) with $\Delta D_{c}=D_{c}(\tau)-D_{c}(0)$ and the symbols are obtained via numerical integrations of Eqs. (3). (b) The symmetric phase at high $D$ is represented by the quadratic free energy and the spontaneous symmetry breaking phase at low $D$ by the double well. (c) Here $P[\phi]$ is obtained through numerical integration of Eqs. (3).

and the only real and physical solution is

$$
\begin{aligned}
& D_{c}(\tau)= \frac{1}{\epsilon}\left[\frac{\lambda}{\gamma}+\gamma-\alpha\right], \quad \epsilon \equiv 3 a_{0}^{3} \tau^{2}, \\
& \lambda \equiv a_{0}^{4} \tau^{2}+18 a_{0}^{3} b \tau^{3}, \quad \alpha \equiv 2 a_{0}^{2} \tau-3 a_{0}^{3} D_{0} \tau^{2}, \\
& \gamma \equiv \\
&\left.\Delta \frac{2 a_{0}^{6} \tau^{3}-108 a_{0}^{5} b \tau^{4}+162 a_{0}^{6} b D_{0} \tau^{5}+\Delta}{2}\right]^{1 / 3}, \\
& \Delta \equiv \\
& \\
&-4\left(2 a_{0}^{6} \tau^{3}-108 a_{0}^{5} b \tau^{4}+162 a_{0}^{6} b D_{0} \tau^{5}\right)^{2}
\end{aligned}
$$

Here $D_{c}(\tau)$ increases for small $\tau$, reaches its maximum value $D^{*}$ at $\tau^{*}$, and decreases to $D_{0}$ for $\tau \rightarrow \infty$. As a consequence, the phase diagram in the $(\tau, D)$ plane is reentrant. This is shown in Fig. 1(b), where the LG free energy is plotted for increasing $\tau$ for $D_{0}<D<D^{*}$. The LG free energy develops a double well for $\tau_{-}<\tau<\tau_{+}$(magenta and yellow curves), with $\tau_{ \pm}$the solutions of $D=D_{c}(\tau)$. For $\tau<\tau_{-}$or $\tau>\tau_{+}$ the system is in the symmetric phase (blue and red curves, respectively).

It is worth noting that a reentrant behavior of the Boyle line has been observed in the virial series of many mutually interacting particles in the presence of correlated noise [19]. The small $\tau$ behavior indicates that memory effects in the dynamics raise the critical effective temperature, suggesting that the activity promotes criticality.

In order to compare the analytical expression for $D_{c}(\tau)$ with the true order parameter dynamics, we have solved numerically the nonequilibrium dynamics. Equations (3) have been numerically integrated for $N_{t}=10^{6}$ steps with $\Delta t=$ $10^{-3}$. The parameters of the model are $a_{0}=4, D_{0}=1,2$, and $b=1$. From the trajectories $\varphi(t)$ we have computed $P[\varphi]=$ $\langle\delta[\varphi(t)-\varphi]\rangle_{t, \varphi(0)}$, where the angular brackets indicate the average over both the trajectories and the initial condition. The critical point has been obtained by fitting the histogram of $P[\varphi]$ to $f(x)=A \exp \left(-\tilde{a} \varphi^{2}-\tilde{b} \varphi^{4}\right)$. We have considered the average over $5 \times 10^{2}$ initial conditions. The resulting $P_{s t}[\varphi]$ for $\tau=0.1$ is shown in Fig. 1(c). As one can see in Fig. 1(a), the theoretical curve $\Delta D_{c} / D_{0}$, with $\Delta D_{c}=D_{c}(\tau)-$ $D_{c}(0)$, reproduces very well the numerical data in a wide range of $\tau$.

For small $\tau$ we can approximate $\ln \Gamma(\varphi) \sim D \tau \partial_{\varphi}^{2} H$, obtaining an effective $\varphi^{6}$ theory. It is well known in the literature that $\varphi^{6}$ theory admits a tricritical point where the second-order phase transition changes in a first-order phase transition [30]. However, in our model the tricritical point is located in an unphysical region. The effective Hamiltonian reads

$$
\begin{aligned}
H_{\mathrm{eff}}^{\text {small }}[\varphi] & =\frac{\tilde{a}}{2} \varphi^{2}+\frac{\tilde{b}}{4} \varphi^{4}+\frac{\tilde{c}}{6} \varphi^{6}, \\
\tilde{a} & \equiv a(1+a \tau)-6 D b \tau, \\
\tilde{b} & \equiv b+4 a b \tau, \\
\tilde{c} & \equiv 3 b^{2} \tau .
\end{aligned}
$$

In this case, the critical line $D_{c}^{\text {small }}$ is given by $\tilde{a}=0$ and satisfies

$$
a(1+\tau a)-6 D \tau b=0 .
$$

Along $D_{c}^{\text {small }}, \varphi_{0}$ behaves like $\varphi_{0} \sim\left(D-D_{c}\right)^{\beta}$ with $\beta=$ $1 / 2$, i.e., the classical mean-field value for the exponent $\beta$ [14].

\section{NUMERICAL SIMULATIONS}

Now we compare the mean-field picture with its finitedimensional counterpart. With this aim, we have performed numerical simulations of $N$ spherical particles interacting through $\phi(r)=4 e_{0}\left[(r / \sigma)^{-12}-(r / \sigma)^{-6}\right]$ in two- $(d=2)$ and three- $(d=3)$ dimensional boxes of side $L$ with periodic boundary conditions. The density of the system is $\rho_{m}=N / L^{d}$. The energy is measured in the unit $e_{0}$ and the density in the 

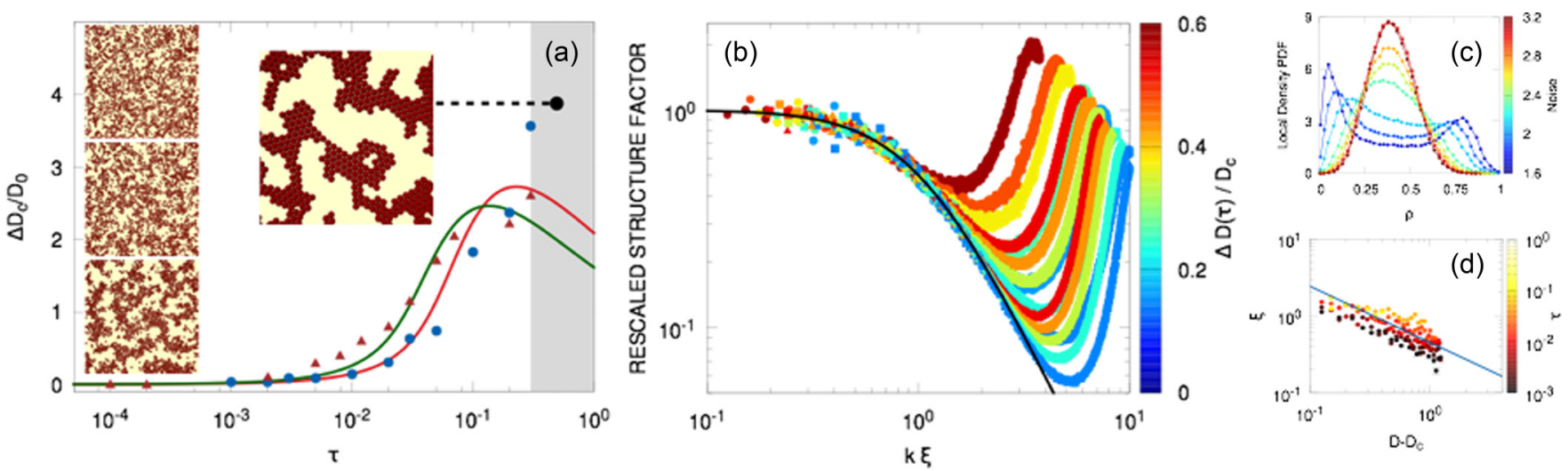

FIG. 2. Numerical simulations. (a) Blue circles and brown triangles are the critical points of the active Lennard-Jones fluid in two and three dimensions, respectively. The solid lines are the fit of the data with Eq. (11). The snapshots on the left show two-dimensional numerical simulations approaching $D_{c}(\tau)$ (from top to bottom) for $\tau=3 \times 10^{-3}$. Cluster crystallization occurs in the gray region. The snapshot on the right shows crystal clusters for $\tau=1.0$. (b) Rescaled structure factor $S(k \xi)$ in two dimensions for $D>D_{c}$ and $\tau=10^{-3}, 10^{-2}, 10^{-1}$ (circles, squares, and triangles, respectively). Symbols represent numerical data and the black curve is obtained by fitting to a Lorentzian distribution. (c) Plot of $P_{\ell}(\rho)$ in three dimensions for $\tau=3 \times 10^{-2}$ and $N_{b}=13$; the lines are a guide for the eye. (d) Plot of $\xi$ obtained from fitting $S(k)$ for small $k$ to $A\left[1+(\xi k)^{2}\right]^{-1}$. The blue line is determined from fitting $\xi$ with $\left(D-D_{c}\right)^{-v}$.

unit $\sigma^{-d}$. The microscopical model undergoes a gas-liquid phase separation that is described by a conserved scalar order parameter $\rho_{G}-\rho_{L}$. The nature of the order parameter implies model B dynamics along the phase separation [37]. However, the location of the critical point, i.e., the end point of the phase separation, is a static property of the system. As mentioned earlier, models A and B show the same static properties: We will adopt the mean-field scenario emerging from model A to capture qualitatively the behavior of the critical line in finite dimension.

The self-propulsion is modeled by means of a random driving force exponentially correlated in time. The equation of motion of the particle $i$, with $i=1, \ldots, N$, is

$$
\dot{\mathbf{r}}_{i}=\mathbf{f}_{i}-\mu \sum_{j \neq i} \phi^{\prime}\left(r_{i j}\right) \mathbf{r}_{i j} / r_{i j},
$$

where $\mu=1$ is the mobility, $\mathbf{r}_{i j}=\mathbf{r}_{i}-\mathbf{r}_{j}$, and $r_{i j} \equiv\left|\mathbf{r}_{i j}\right|$. The noise satisfies $\left\langle f_{i}^{\alpha}\right\rangle=0$ and $\left\langle f_{i}^{\alpha}(t) f_{j}^{\beta}(s)\right\rangle=2 D \delta_{i j} \delta_{\alpha \beta} e^{-|t-s| / \tau} / \tau$, where the greek symbols indicate the Cartesian components. In this picture the external parameters $D$ and $\tau$ can be independently varied as well as in the Landau model previously considered. For $\tau=0$ one recovers the Lennard-Jones (LJ) fluid in the Brownian regime. We study the system close to the LJ critical density $\rho_{c} \sim 0.4$ ( $N=2500$ in two dimensions and $N=8000$ in three dimensions). Moreover, performing simulations at different values of $\rho_{m}{ }^{2}$ we have checked that the value $\rho_{c}$ does not vary with $\tau$, in the range of the motility parameters explored. The critical values $D_{c}(\tau)$ have been evaluated by looking at the intersection points of the Binder cumulant $U_{\ell}=1-\left\langle\delta \rho^{4}\right\rangle_{\ell} / 3\left\langle\delta \rho^{2}\right\rangle_{\ell}^{2}[38]$ at different $\ell$, with $\delta \rho=\rho-$ $\langle\rho\rangle$, where the average is defined as $\langle\mathcal{O}\rangle_{\ell}=\int d \rho P_{\ell}(\rho) \mathcal{O}(\rho)$. The block density distribution function $P_{\ell}(\rho) \equiv\left\langle\delta\left(\rho-\rho_{k}\right)\right\rangle$ is obtained by dividing the simulation box with linear size $L$ in $N_{b}$ cells of size $\ell=L / N_{b}$ and coarse-grained density $\rho_{k}$ with $k=1, \ldots, N_{b}[39,40]$. The behavior of $P_{\ell}(\rho)$ in three dimensions approaching the transition is shown in Fig. 2(c). In order to evaluate the intersection of $U_{\ell}$ as a function of $D$, we have performed simulations of 52 different $D$ for each value of $\tau$.

The resulting phase diagram is shown in Fig. 2(a) with snapshots of the two-dimensional simulations (bottom left inset). The solid lines are obtained by fitting the data with Eq. (11), leaving both $a_{0}$ and $b$ as free parameters. As one can see, for small $\tau$ the theory reproduces quite well the numerical data. However, with the model simulated we cannot probe the regime at larger $\tau$ values since crystallization occurs at $\tau>0.3$ [the gray area in Fig. 2(a)]. In order to prevent crystallization, one can introduce frustration in the microscopical model by considering, for instance, a binary mixture [41]. Hence, the existence of the reentrance in the activity-noise phase diagram remains an open question. It is worth noting that our starting point is a $\varphi^{4}$ theory. In such a case we cannot describe a phase diagram that shows both gas-liquid and gas-crystal phase transitions. Nevertheless, it is possible to generalize our mean-field model by considering a different field theory in order to take into account the presence of a crystal phase [14].

In the crystal regime, the nucleated liquid droplets rearrange into small crystal clusters. The presence of the crystal clusters is evident in the snapshot shown in Fig. 2(a), right.

\section{GAUSSIAN FLUCTUATIONS}

Let us discuss the effect of the correlated noise on the Gaussian fluctuations around the mean field [14]. The

\footnotetext{
${ }^{2}$ In two dimensions $\tau=0,10^{-3}, 3 \times 10^{-3}, 5 \times 10^{-5}, 10^{-1}, 3 \times$ $10^{-1}, 5 \times 10^{-1}, 6 \times 10^{-1}, 0,1,0.3,0.5$. In three dimensions $\tau=0,10^{-4}, 2 \times 10^{-3}, 5 \times 10^{-3}, 8 \times 10^{-3}, 10^{-2}, 2 \times 10^{-2}, 3 \times$ $10^{-2}, 5 \times 10^{-2}, 7 \times 10^{-2}, 0.3,1,10$. We have explored $\rho_{m} \in[0.2,0.7]$.
} 
Hamiltonian in $d$ dimensions is

$$
H_{G}[\varphi(\mathbf{x}, t)]=\frac{1}{2} \int d^{d} x\left\{[\nabla \varphi(\mathbf{x}, t)]^{2}+a \varphi(\mathbf{x}, t)^{2}\right\} .
$$

We will consider both model $\mathrm{A}$ and model $\mathrm{B}$ dynamics with exponentially correlated noise. We can rewrite Eqs. (3) including the spatial dependence in a compact way as follows:

$$
\begin{aligned}
& \partial_{t} \varphi(\mathbf{x}, t)=-(i \nabla)^{2 \psi}\left(\frac{\delta H_{G}}{\delta \varphi(\mathbf{x}, t)}\right)+(-\nabla)^{\psi} \theta(\mathbf{x}, t), \\
& \partial_{t} \theta(\mathbf{x}, t)=-\frac{\theta(\mathbf{x}, t)}{\tau}+\frac{D^{1 / 2}}{\tau} \eta(\mathbf{x}, t) .
\end{aligned}
$$

The exponent $\psi$ is 0 (model A) or 1 (model B). The noise is white $\langle\eta(\mathbf{x}, t)\rangle=0$ and $\delta$ correlated $\langle\eta(\mathbf{x}, t) \eta(\mathbf{y}, s)\rangle=$ $2 \delta(\mathbf{x}-\mathbf{y}) \delta(t-s)$.

Now we introduce the spatial Fourier transform of a field $\phi(\mathbf{x})$ as

$$
\phi_{\mathbf{k}}=\frac{1}{(2 \pi)^{d}} \int d^{d} x e^{-i \mathbf{x} \cdot \mathbf{k}} \phi(\mathbf{x}) .
$$

We can perform the spatial Fourier transform of $\varphi(\mathbf{x}, t), \theta(\mathbf{x}, t)$, and $\eta(\mathbf{x}, t)$, obtaining the time evolution of the $k$ th Fourier component of $\varphi$ in the UCNA, i.e., considering $\partial_{t}^{2} \varphi_{k}=0$, which is governed by

$$
\begin{aligned}
\partial_{t} \varphi_{k} & =-\Gamma_{k} \varphi_{k}+\mathcal{D}_{k} \eta_{k}, \quad \Gamma_{k} \equiv k^{2 \psi} \frac{k^{2}+a}{1+\tau\left(k^{2}+a\right)}, \\
\mathcal{D}_{k} & \equiv(i k)^{\psi} \frac{D^{1 / 2}}{1+\tau\left(k^{2}+a\right)},
\end{aligned}
$$

where $k \equiv|\mathbf{k}|$. Again, the noise $\eta_{k}$ is white $\left\langle\eta_{k}\right\rangle=0$ and $\delta$ correlated $\left\langle\eta_{k}(t) \eta_{q}(s)\right\rangle=2 \delta_{k q} \delta(t-s)$. We can estimate the critical slowing down exponent by averaging Eq. (18) over the noise. It follows that $\left\langle\varphi_{k}(t)\right\rangle \propto \exp \left(-t / \tau_{k}\right)$, with $\tau_{k}=\Gamma_{k}^{-1}$. Introducing $\xi^{2}=a^{-1}$, we can write the relaxation time of the mode $k$ as $\tau_{k}=\xi^{z} f\left(k \xi, \tau \xi^{-2}\right)$. The value of the dynamical critical exponent $z=2(1+\psi)$ turns out to be the same as in the case of equilibrium dynamics [37].

From Eq. (18) we can compute the stationary fluctuations $\left\langle\left|\varphi_{k}\right|^{2}\right\rangle=\xi^{2} g(k \xi, \tau)$ with

$$
g(k \xi, \tau)=\frac{D}{\left(k^{2} \xi^{2}+1\right)\left[1+\tau(k \xi)^{2 \psi} \xi^{-2(1+\psi)}\left(k^{2} \xi^{2}+1\right)\right]} .
$$

When $k \rightarrow 0,\left\langle\left|\varphi_{k}\right|^{2}\right\rangle$ diverges as $\xi=a^{-v}$, with the classical value $v=1 / 2$. From Eq. (19) it follows that, above the transition, the static structure factor $S(k)$ for small $k$ is well described by the usual OZ expression $S(k) \sim\left(k^{2} \xi^{2}+1\right)^{-1}$. In Fig. 2(b) we show the rescaled $S(k)$ in two dimensions for $\tau=10^{-3}, 10^{-2}, 10^{-1}$ and $D>D_{c}(\tau)$. According to the OZ expression, the rescaled $S(k)$ overlaps on the same master curve. Moreover, as shown in Fig. 2(d), $\xi$ follows a power law $\left(D-D_{c}\right)^{-v_{N}}$, with $v_{N}=0.73$ for $N=2500$. The value of the exponent does not depend on $\tau$, i.e., as predicted by the mean-field picture. Since this is in finite dimension, it is different from the classical value $1 / 2$ [14]. It is worth noting that $v$ has been evaluated for $N=2500$. In order to estimate the critical exponent $v$ in the thermodynamic limit, we should take into account the finite-size correction to $D_{c}$, i.e.,
$D_{c}(L)=D_{c}^{\infty}+a L^{-b}$ [42]. This aspect is beyond the scope of the present paper.

\section{CONCLUSION}

In this article we have posed a fundamental question about the influence of self-propulsion on order-disorder transitions. By means of the UCNA, we have recast the nonequilibrium dynamics of models A and B in the presence of correlated noise in an effective equilibrium theory. In this way we have extended the static picture of the Landau theory of critical phenomena to order-disorder transitions in the presence of exponentially correlated noise. The location of the critical point is a nonuniversal quantity because it depends on the correlation time of the noise $\tau$. Through the effective equilibrium theory we have computed analytically the critical line $D_{c}(\tau)$, i.e., the shift in critical temperature due to the activity. Considering the numerical solution of the nonequilibrium dynamics of the order parameter in zero dimension, we have observed good agreement between $D_{c}(\tau)$ and the critical points obtained numerically. Moreover, the effective theory suggests that the out-of-equilibrium dynamics does not change the universality class. This finding is in agreement with previous studies on Ising-like nonequilibrium models [43-45], where it has been observed that the absence of detailed balance on the microscopic scale does not change the universality class of the Ising model.

By performing numerical simulations in two and three dimensions of active LJ fluid driven by the OUP, we have obtained that the mean-field scenario can be used to describe the behavior of the critical line in the small $\tau$ regime. However, the reentrant behavior predicted by the mean-field scenario does not occur in the considered microscopical model. In particular, the numerical simulations show a gas-crystal phase transition at larger $\tau$ that cannot be captured by the theory. With the numerical data presented in this paper we can conclude that, for small $\tau$ and independently of density, the out-of-equilibrium dynamics gives rise to a second-order phase transition that has the same properties as its equilibrium counterpart, i.e., $\tau=0$.

Finally, we have evaluated the Gaussian fluctuations approaching the critical point from the disordered phase. According to the theory, we have demonstrated that the static structure factor at low $k$ is well described by the OZ expression. Here we have considered a $\varphi^{4}$ scalar field theory, however, our approach can be generalized to other field theories in order to study the properties of different universality classes under the effect of self-propulsion.

\section{ACKNOWLEDGMENTS}

We thank M. Cristina Marchetti for her critical reading of an early version of the manuscript. M.P. was supported by the Simons Foundation Targeted Grant in the Mathematical Modeling of Living Systems No. 342354 and by the Syracuse Soft Matter Program. C.M. was supported by the European Research Council under the European Union's Seventh Framework Programme (FP7/2007-2013)/ERC Grant No. 307940. N.G. acknowledges support from MIUR ("Futuro in Ricerca" ANISOFT/RBFR125H0M) and from the European Research Council (ERC Consolidator Grant No. 681597, MIMIC). 
[1] M. C. Marchetti, J. F. Joanny, S. Ramaswamy, T. B. Liverpool, J. Prost, M. Rao, and R. A. Simha, Rev. Mod. Phys. 85, 1143 (2013).

[2] A. Cavagna and I. Giardina, Annu. Rev. Condens. Matter Phys. 5, 183 (2014).

[3] T. Vicsek and A. Zafeiris, Phys. Rep. 517, 71 (2012).

[4] M. E. Cates, Rep. Prog. Phys. 75, 042601 (2012).

[5] T. Vicsek, A. Czirok, E. Ben-Jacob, I. Cohen, and O. Shochet, Phys. Rev. Lett. 75, 1226 (1995).

[6] H. Chaté, F. Ginelli, G. Grégoire, F. Peruani, and F. Raynaud, Eur. Phys. J. B 64, 451 (2008).

[7] A. Baskaran and M. C. Marchetti, Proc. Natl. Acad. Sci. USA 106, 15567 (2008).

[8] G. S. Redner, A. Baskaran, and M. F. Hagan, Phys. Rev. E 88, 012305 (2013).

[9] J. Tailleur and M. E. Cates, Phys. Rev. Lett. 100, 218103 (2008).

[10] S. Henkes, Y. Fily, and M. C. Marchetti, Phys. Rev. E 84, 040301 (2011).

[11] L. Berthier and J. Kurchan, Nat. Phys. 9, 310 (2013).

[12] D. Bi et al., Nat. Phys. 11, 1074 (2015).

[13] D. Bi, X. Yang, M. C. Marchetti, and M. L. Manning, Phys. Rev. X 6, 021011 (2016).

[14] M. Le Bellac, Quantum and Statistical Field Theory (Clarendon, Oxford, 1991).

[15] C. Maggi, U. Marini Bettolo Marconi, N. Gnan, and R. Di Leonardo, Sci. Rep. 5, 10742 (2015).

[16] T. F. F. Farage, P. Krinninger, and J. M. Brader, Phys. Rev. E 91, 042310 (2015).

[17] É. Fodor, C. Nardini, M. E. Cates, J. Tailleur, P. Visco, and F. van Wijland, Phys. Rev. Lett. 117, 038103 (2016).

[18] G. Szamel, E. Flenner, and L. Berthier, Phys. Rev. E 91, 062304 (2015).

[19] U. Marini Bettolo Marconi and C. Maggi, Soft Matter 11, 8768 (2015).

[20] U. Marini Bettolo Marconi, N. Gnan, M. Paoluzzi, C. Maggi, and R. Di Leonardo, Sci. Rep. 6, 23297 (2016).

[21] U. Marini Bettolo Marconi, M. Paoluzzi, and C. Maggi, Mol. Phys. 114, 1 (2016).
[22] P. Jung and P. Hänggi, Phys. Rev. A 35, 4464 (1987).

[23] P. Hänggi and P. Jung, Adv. Chem. Phys. 89, 239 (1995).

[24] Y. Fily and M. C. Marchetti, Phys. Rev. Lett. 108, 235702 (2012).

[25] N. Koumakis, C. Maggi, and R. Di Leonardo, Soft Matter 10, 5695 (2014).

[26] C. Maggi, M. Paoluzzi, N. Pellicciotta, A. Lepore, L. Angelani, and R. Di Leonardo, Phys. Rev. Lett. 113, 238303 (2014).

[27] X. L. Wu and A. Libchaber, Phys. Rev. Lett. 84, 3017 (2000).

[28] G. Szamel, Phys. Rev. E 90, 012111 (2014).

[29] K. G. Wilson, in The Renormalization Group and Critical Phenomena, Nobel Lectures 1981-1990, edited by G. Ekspöng (World Scientific, Singapore, 1993).

[30] J. Zinn-Justin, Quantum Field Theory and Critical Phenomena (Clarendon, Oxford, 2002).

[31] P.-G. de Gennes, The Physics of Liquid Crystals (Clarendon, Oxford, 1974).

[32] V. Narayan, S. Ramaswamy, and N. Menon, Science 317, 105 (2007).

[33] H. Chaté, F. Ginelli, and R. Montagne, Phys. Rev. Lett. 96, 180602 (2006).

[34] A. Baskaran and M. C. Marchetti, Phys. Rev. E 77, 011920 (2008).

[35] E. Bertin et al., New J. Phys. 15, 085032 (2013).

[36] S. P. Thampi, R. Golestanian, and J. M. Yeomans, Phys. Rev. E 90, 062307 (2014).

[37] P. C. Hohenberg and B. I. Halperin, Rev. Mod. Phys. 49, 435 (1977).

[38] K. Binder, Z. Phys. B 43, 119 (1981).

[39] M. Rovere, D. W. Heermann, and K. Binder, Europhys. Lett. 6, 585 (1998).

[40] M. Rovere, D. W. Heermann, and K. Binder, J. Phys.: Condens. Matter 2, 7009 (1999).

[41] W. Kob and H. C. Andersen, Phys. Rev. Lett. 73, 1376 (1994).

[42] M. Palassini and S. Caracciolo, Phys. Rev. Lett. 82, 5128 (1999).

[43] J. F. F. Mendes and M. A. Santos, Phys. Rev. E 57, 108 (1998).

[44] M. J. de Oliveira, J. Stat. Phys. 66, 273 (1992).

[45] C. H. Bennett and G. Grinstein, Phys. Rev. Lett. 55, 657 (1985). 